\title{
INFLUENCE OF ACTIVE NITROGEN SPECIES ON THE NITRIDATION RATE OF SAPPHIRE
}

\author{
A.J. Ptak ${ }^{*}$, K.S. Ziemer ${ }^{* *}$, M.R. Millecchia ${ }^{*}$, C.D. Stinespring ${ }^{* *}$, and T.H. Myers ${ }^{*}$, \\ * Department of Physics, ${ }^{* *}$ Department of Chemical Engineering, West Virginia University, \\ Morgantown, WV 26506; ${ }^{\dagger}$ tmyers@wvu.edu
}

Cite this article as: MRS Internet J. Nitride Semicond. Res. 4S1, G3.10 (1999)

\begin{abstract}
The operating regimes of two rf-plasma sources, an Oxford CARS-25 and an EPI Unibulb, have been extensively characterized. By changing the exit aperture configuration and using an electrostatic deflector, the Oxford source could produce either primarily atomic nitrogen, atomic nitrogen mixed with low energy ions, or a large flux of higher energy ions (>65 $\mathrm{eV}$ ) as the active species in a background of neutral molecular nitrogen. The EPI source produced a significant flux of metastable molecular nitrogen as the active species with a smaller atomic nitrogen component. Nitridation of sapphire using each source under the various operating conditions indicate that the reactivity was different for each type of active nitrogen. Boron contamination originating from the pyrolytic boron nitride plasma cell liner was observed.
\end{abstract}

\section{INTRODUCTION}

GaN has been grown by many techniques, each with its own form of active nitrogen species. Molecular beam epitaxy (MBE), using either electron cyclotron radiation (ECR) or radio frequency (rf) plasma sources, represents a particularly complex case since these sources produce ions of various energies, atoms, and potentially, nitrogen molecules in a metastable state. The reactivity of the individual species may play a crucial role in growth kinetics, particularly in the formation of point defects. One measure of the reactivity of a given species is indicated by its efficiency at the nitridation of sapphire, a common step in the nucleation process of $\mathrm{GaN}$. The different reactivity of the various species is evident from the wide range of nitridation times observed $[1,2,3,4]$. We report the results of a study determining the active species from two rf-plasma sources under different operating conditions, and assess the relative reactivity of the various nitrogen species based on nitridation rates for sapphire.

\section{RF-PLASMA SOURCE CHARACTERIZATION}

Two rf-plasma sources were used to produce various species of nitrogen for the nitridation of sapphire. These sources were an Oxford Applied Research (Oxfordshire, England) CARS-25 source and an EPI Vacuum Products (St. Paul, MN) Unibulb source. The Oxford source featured a removable pyrolytic boron nitride (PBN) aperture plate allowing use of different hole configurations. We investigated plates with 1, 9, 37, and 255 holes. As the number of holes increased, the size of the holes was decreased proportionately in order to maintain the overall conductance of the plate. The EPI source contained the standard Unibulb configuration with a 400-hole aperture resulting in an approximately $50 \%$ increase in conductance.

The sources were mounted in a UHV chamber in direct line of sight to an Extrel quadrupole mass spectrometer for characterization. The mass spectrometer's repeller grid was biased separately to allow a determination of ion energies in the flux. Additionally, a stainless 
steel electrostatic deflector plate was positioned below the nitrogen source that could be biased to deflect ions out of the flux. The sources were operated under conditions producing a maximum growth rate for $\mathrm{GaN}$ in previous $\mathrm{MBE}$ growth experiments. Characterization results are summarized in Table I.

The Oxford source with the single-hole aperture produced a significant flux of highenergy ions, both molecular and atomic, similar to an ECR source [5]. The large, high-energy ion flux precluded accurate measurement of a neutral atomic nitrogen concentration. Reducing the aperture hole size also reduced the ion content in the flux as well as the total ion energy, probably due to wall interactions, as indicated in Table I. In essentially all the cases, the concentration of atomic nitrogen ions was two to three times that of molecular ions. The atomic nitrogen ions were also typically 5 to $10 \mathrm{eV}$ more energetic, with both distributions peaked near the maximum energies. The maximum ion energy range listed in Table I is representative of the difference between molecular and atomic ionic species. Use of the 9, 37, or 255-hole aperture led to a varying mixture of atomic nitrogen and ions. Similar results have been reported for apertures used on an ECR source.[6] Atomic nitrogen accounted for 2-6\% of the total nitrogen flux, with the ion content reduced to less than $0.1 \%$. Another source parameter, the incorporation efficiency, was determined by measuring the nitrogen actually incorporated in $\mathrm{GaN}$ based on the growth rates from separate MBE growth experiments compared to the total nitrogen impinging on the growing layer. Measured growth rates were the right magnitude to be due to the atomic nitrogen, and too large to be due to the ions. While the incorporation efficiency scaled well with atomic nitrogen concentrations, it is clear that a significant fraction of the atomic nitrogen is not incorporated into the growing layer.

Several significant differences were observed for the EPI source. First, the total ion content was significantly reduced, to less than $3 \times 10^{-5}$ percent of the total flux, with the remaining ions having energies less that $3 \mathrm{eV}$. Second, the incorporation efficiency was significantly larger, particularly when considering the measured atomic nitrogen concentration. During the characterization of the plasma sources, the mass spectrometer ionizer energy was set high enough to ionize both molecular and atomic nitrogen (typically $>15 \mathrm{eV}$ ), but too low to crack a significant portion of molecular nitrogen into atomic nitrogen $(>28 \mathrm{eV})$. During the EPI source characterization, considerable molecular nitrogen ions were produced with an ionizer

Table I. Results of rf-plasma source characterization.

\begin{tabular}{|c|c|c|c|c|c|c|}
\hline \multirow[b]{2}{*}{ SOURCE } & \multicolumn{2}{|c|}{ APERTURE } & \multirow[b]{2}{*}{$\begin{array}{c}\text { Ion } \\
\text { Content } \\
(\%)\end{array}$} & \multirow[b]{2}{*}{$\begin{array}{c}\text { Max. Ion } \\
\text { Energy } \\
(\mathrm{eV})\end{array}$} & \multirow[b]{2}{*}{$\begin{array}{c}\text { Atom } \\
\text { Efficiency } \\
(\%)^{\mathrm{a}}\end{array}$} & \multirow{2}{*}{$\begin{array}{c}\mathrm{GaN} \\
\text { Incorporation } \\
\text { Efficiency } \\
(\%)^{\mathrm{b}}\end{array}$} \\
\hline & $\begin{array}{c}\text { Hole } \\
\text { Diameter } \\
(\mathrm{mm})\end{array}$ & $\begin{array}{l}\text { Number } \\
\text { of Holes }\end{array}$ & & & & \\
\hline \multirow{4}{*}{$\begin{array}{l}\text { OXFORD } \\
\text { CARS-25 }^{\text {d }}\end{array}$} & 3.0 & 1 & 5 & $>65$ & c & \\
\hline & 1.0 & 9 & 0.1 & $40-50$ & $5-6$ & 0.48 \\
\hline & 0.5 & 37 & 0.05 & $15-25$ & $3-4$ & 0.32 \\
\hline & 0.2 & 255 & 0.03 & $8-12$ & $2-3$ & 0.23 \\
\hline EPI Unibulb & 0.2 & 400 & $<3 \times 10^{-5}$ & $<3$ & $2-3$ & 7.2 \\
\hline
\end{tabular}

${ }^{\mathrm{a}}$ atomic $\mathrm{N}$ out/total $\mathrm{N}$ into source $\mathrm{x} 100 \%{ }^{\mathrm{b}} \mathrm{N}$ incorporated in $\mathrm{GaN} /$ total $\mathrm{N}$ into source times $100 \%{ }^{\mathrm{c}}$ not measurable ${ }^{\mathrm{d}} 600 \mathrm{~W}, 6 \mathrm{SCCM}^{\mathrm{e}}$ 600W, 2 SCCM 


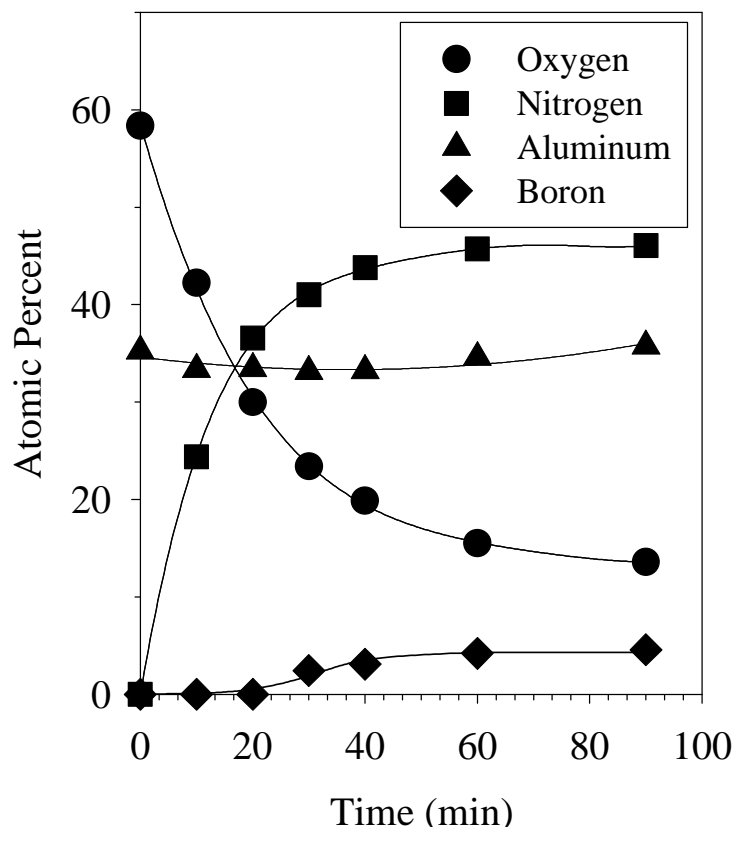

Figure 1. Auger analysis of Al, O, N, and B during nitridation of $\mathrm{Al}_{2} \mathrm{O}_{3}$ using the Oxford source with a single hole aperture. The solid lines are guides to the eyes. energy approximately $6 \mathrm{eV}$ lower than normally necessary to ionize molecular nitrogen. This energy difference corresponds to the $A^{3} \Sigma_{u}^{+}$metastable state of the nitrogen molecule, and indicates this source produces a significant amount of nitrogen metastables. The presence of the metastables complicated the source characterization somewhat, but we were able to determine an atomic nitrogen efficiency of about 2-3\%. This source has proven quite effective at incorporation of nitrogen in $\mathrm{GaN}$, with growth rates at least an order of magnitude too large to be due to the atomic nitrogen based on comparison with the Oxford source. Since the ion content is negligible, the most likely active nitrogen species remaining is the molecular nitrogen metastable.

\section{NITRIDATION OF SAPPHIRE}

Nitridation experiments were performed in the same chamber as the rf-plasma source characterization using a heated sample stage. Nitridation at low temperature was chosen to accentuate differences in the reactivity of the various species. Nitridation did not occur at room temperature for any source configuration, but proceeded at a reasonable rate at $400^{\circ} \mathrm{C}$, which was used for all the nitridation experiments. The nitridation chamber was connected to a separate chamber where Auger measurements could be performed using a Phi 545 Scanning AES Microprobe with Model 110A Cylindrical Electron Optics. Scans were performed with a 3 $\mathrm{keV}$ incident electron beam, a beam current of $2 \mu \mathrm{A}$ and a nominal spot size of $3 \mu \mathrm{m}$.

Figure 1 details Auger analysis made during a nitridation run using the Oxford source with the single-hole aperture. The analysis uses elemental sensitivity factors [7], and is representative of the number of atoms of each species present within the Auger sampling depth. All of the nitridations performed with the Oxford source gave similar results, but with different time scales for differing conditions. Yeadon et al. have shown [8] that $\mathrm{AlN}$ and $\mathrm{Al}_{2} \mathrm{O}_{3}$ are immiscible and nitridation proceeds by the formation of an $\mathrm{AlN}$ overlayer on the $\mathrm{Al}_{2} \mathrm{O}_{3}$. Atomic force microscopy of a sapphire surface nitrided under similar conditions to that of the sample of Fig. 1 indicated a flat, featureless surface. Thus in order to quantitatively compare the nitridation rates, we used a standard model [9] for oxygen signal attenuation by an AlN overlayer normalized by the total Al signal to estimate AlN thickness. The model used a measured oxygen sensitivity factor relative to aluminum from $\mathrm{Al}_{2} \mathrm{O}_{3}$ and electron inelastic mean free paths based on the model by Tanuma et al. [10] The relative shift in $\mathrm{Al}$ signal strength between $\mathrm{Al}_{2} \mathrm{O}_{3}$ and AlN has been taken into account in the model. The model did not include backscatter correction factors, and so the calculated thickness values should be treated as approximate. 


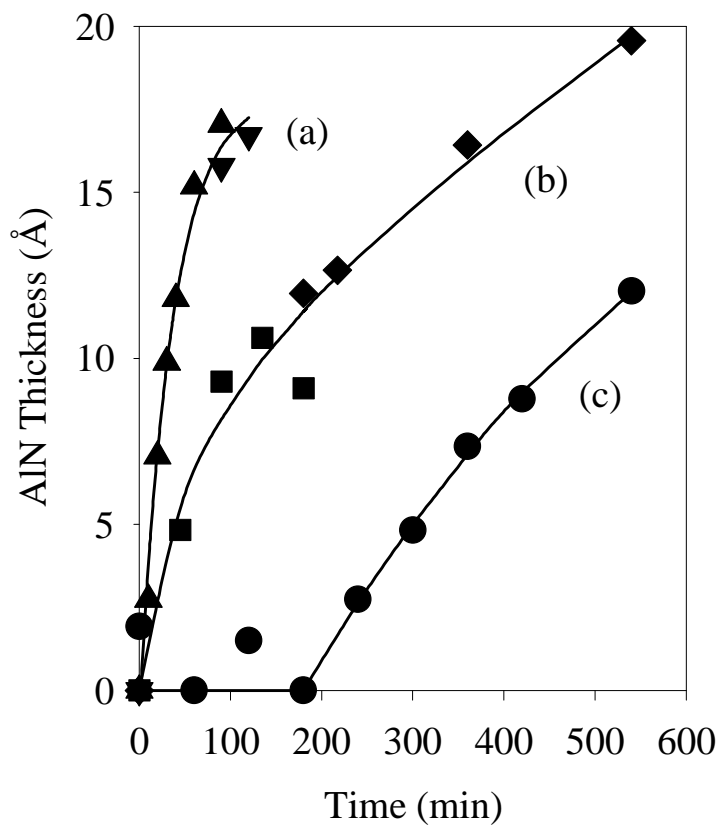

Figure 2. AlN overlayer thickness as a function of time for (a) $\triangle$ and $\nabla$, single-hole aperture; (b) $\square$ and $\diamond$, 9-hole aperture with ions deflected; and (c) o, 9-hole aperture without ion deflection. The solid lines are guides to the eye.

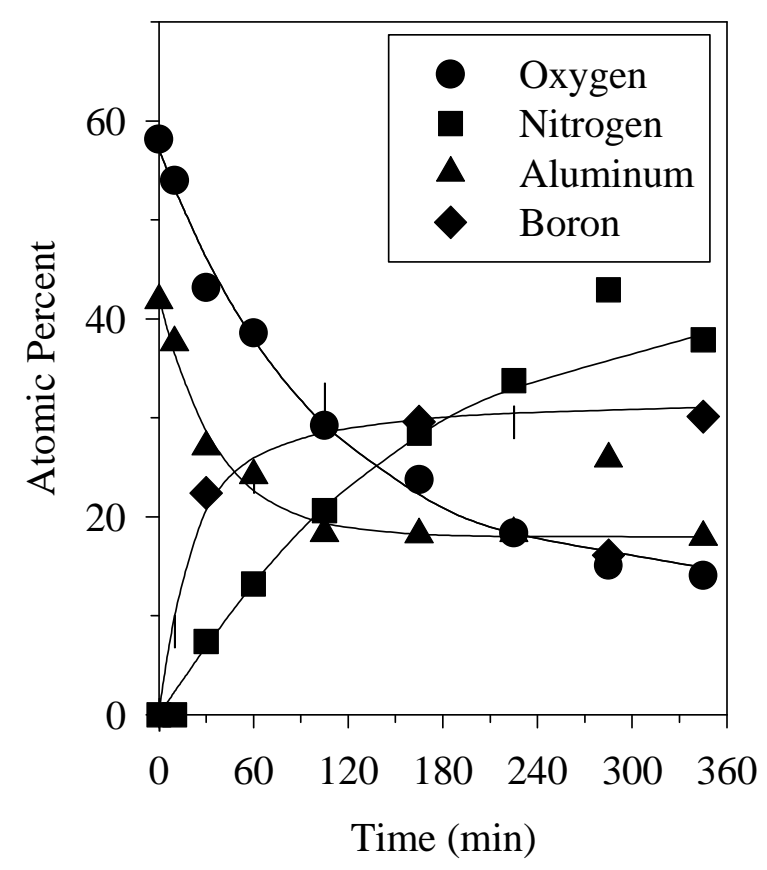

Figure 3. Auger analysis of $\mathrm{Al}, \mathrm{O}, \mathrm{N}$, and $\mathrm{B}$ during nitridation using the EPI source.
Figure 2 summarizes AlN overlayer thickness as a function of time for three configurations of the CARS-25 source. Fig. 2(a) corresponds to the nitridation of two samples using the single-hole aperture, with a large flux (5\%) of high-energy molecular and atomic nitrogen ions. This condition is similar to an ECR source, and results in a relatively rapid nitridation with a rate of about $0.3 \AA / \mathrm{min}$ for the first $10 \AA$. Fig. 2(c) is representative of the 9-hole aperture results. There appears to be an initiation stage lasting approximately 180 minutes, followed by AlN formation at a rate of about $0.03-0.04 \AA / \mathrm{min}$. The active flux from this source configuration is a mixture of both atomic nitrogen $(5-6 \%)$ and nitrogen ions (0.1\%). Fig. 2(b) represents the same configuration, but now the electrostatic deflector plate was used to remove the ions from the flux. Note the nitridation began immediately, and maintained a rate approximately three times $(0.1 \AA / \mathrm{min})$ that shown in Fig. 2(c). The trends shown in Fig.2 indicate that both energetic nitrogen ions and atomic nitrogen are quite reactive, with some indication that the energetic ions are the more reactive species. This is particularly evident for larger AlN thickness. Of equal interest is that a flux of lower energy ions apparently suppresses the nitridation process. A likely mechanism is that atomic nitrogen is adsorbed on the growing surface in a weakly bound state. The incoming ions (and possibly atoms) interact with this adsorbed nitrogen forming molecular nitrogen that quickly desorbs, removing these atoms from participating in the nitridation process. The initiation stage represents a period where the adsorbed nitrogen is very weakly bound to the sapphire surface and easily removed. Based on the rapid onset of AlN growth once initiated, it is likely that once AlN 


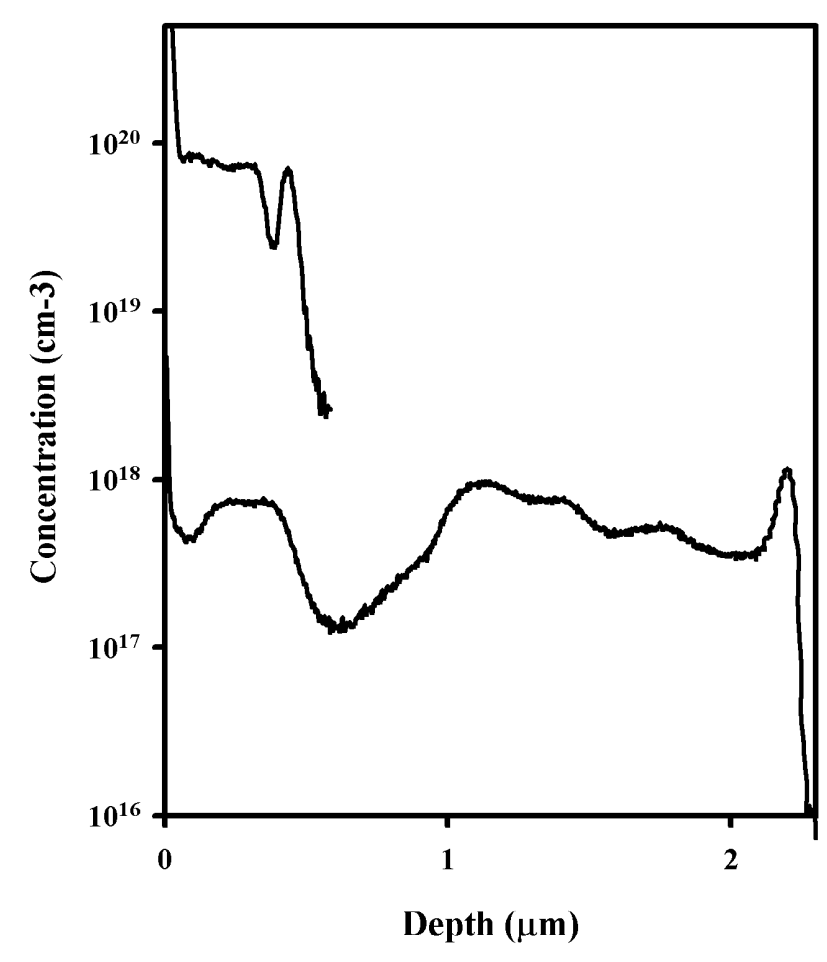

Figure 4. SIMS of B GaN grown using (a) the OXFORD source and (b) the EPI source. is formed, the nitrogen is more strongly bound to the surface and the nitridation process then proceeds.

Figure 3 summarizes Auger analysis of nitridation using the EPI source. The results were substantially different from that observed with the Oxford source. While boron was observed for all nitridation conditions, the Auger analysis indicates significantly more boron on the $\mathrm{Al}_{2} \mathrm{O}_{3}$ surface exposed to the EPI source. The results imply that an overlayer of $\mathrm{BN}$ was formed within the first 20 minutes of nitridation, followed by an apparent cessation of $\mathrm{BN}$ formation. During this initial stage, both the $\mathrm{Al}$ and $\mathrm{O}$ Auger signals reduced in proportion, consistent with $\mathrm{BN}$ overlayer coverage without AlN formation. Surprisingly, both the B and the Al signal then became constant, while the $\mathrm{O}$ signal decreased and the $\mathrm{N}$ signal continued to increase. The latter is suggestive that $\mathrm{AlN}$ is now forming. Since the B signal also remains constant, it is feasible that $\mathrm{Al}_{1-\mathrm{x}} \mathrm{B}_{\mathrm{X}} \mathrm{N}$ may actually be forming. The short predicted mean free paths for $\mathrm{B}(7 \AA)$ and $\mathrm{Al}(6 \AA)$ Auger electrons compared to that of $\mathrm{O}(11 \AA)$ or $\mathrm{N}(14 \AA)$ would result in a constant $\mathrm{B}$ and $\mathrm{Al}$ signal with the others still changing. Assuming that only AlN formation is occurring, modeling interprets an $\mathrm{AlN}$ growth rate during this second stage of $0.07 \AA / \mathrm{min}$, consistent with the atomic nitrogen concentration in the flux coupled with the lack of ions. This latter result may indicate that the nitrogen metastables believed to be present are relatively non-reactive, at least as far as nitridation is concerned. Interpretation of this set of Auger measurements, however, is certainly complicated.

The results depicted in Fig. 3 are suggestive that the flux from the EPI source has a significantly larger B content than the Oxford source, likely due to the degradation of the pyrolytic boron nitride $(\mathrm{PBN})$ liner. In actuality, this result is more indicative of the efficacy of metastable nitrogen for group III-nitride layer growth. Fig. 4 summarizes the B content for two GaN layers measured using secondary ion mass spectrometry (SIMS) (Charles Evans and Associates). Fig. 4(a) represents the B concentration in a layer grown using the Oxford source with the 9-hole aperture, yielding an average B concentration of about $8 \times 10^{19} \mathrm{~cm}^{-3}$. Similar results were obtained for the other aperture sets, and also after replacing the PBN liner in the Oxford source. The EPI source actually emitted significantly less B, with incorporation levels in the mid- $10^{17}$ to $1 \times 10^{18} \mathrm{~cm}^{-3}$ range, as shown by Fig 4(b). The variation in B concentration for each trace corresponds to different source rf-powers, with the lowest corresponding to $200 \mathrm{~W}$ and the highest to $600 \mathrm{~W}$. A similar difference in B incorporation has also been reported to occur with these sources for nitrogen doping of $\mathrm{ZnSe}$ and CdTe [11].

Thus, a likely interpretation of the rapid BN layer formation indicated by the results in Fig. 3 is that $A^{3} \Sigma_{u}^{+}$nitrogen molecules are particularly efficacious for III-N growth. This is indicated both by the rapid $\mathrm{BN}$ overlayer formation, and by the significant increase in nitrogen incorporation efficiency observed for MBE growth. Enhanced growth using nitrogen 
metastables is clearly demonstrated by the large $(\sim 2.3 \mu \mathrm{m} / \mathrm{hr})$ growth rates we have obtained with the EPI rf-plasma source.

\section{CONCLUSIONS}

Nitridation of sapphire was investigated using two rf-plasma sources to determine the reactivity of various types of active nitrogen. The Oxford CARS-25 source, in conjunction with an electrostatic deflector plate, could provide an active nitrogen flux consisting of high energy ionic molecular and atomic nitrogen, a mixture of atomic nitrogen and lower energy ions, or predominantly atomic nitrogen. The EPI Unibulb source produced a significant amount of metastable molecular nitrogen. The nitridation rate for high-energy ions was $\sim 0.3 \AA / \mathrm{min}$ for the first $10 \AA$, about three times faster than nitridation by atomic nitrogen at a similar concentration. The mixed atomic nitrogen/low energy ion condition showed an initiation stage that lasted for $\sim 180$ minutes, followed by nitridation at a rate of $0.03-0.04 \AA / \mathrm{min}$. This initiation stage may be due to formation and desorption of molecular nitrogen from the sapphire surface. Metastable nitrogen does not appear to be very reactive for nitridation, but does appear efficacious for the growth of group III-nitrides. Boron contamination was observed from both sources at levels significant enough to warrant further investigation.

\section{ACKNOWLEDGEMENTS}

This work was supported by ONR Grant N00014-96-1-1008 and monitored by Colin Wood. We would like to acknowledge continuing useful conversations with P. I. Cohen, R. M. Feenstra, N. Newman and C. E. C. Wood.

\section{REFERENCES}

[1] C. Heinlein, J. Grepstad, T. Berge, and H. Riechert, Appl. Phys. Lett. 71, 3, (1997)

[2] C. Heinlein, J. Grepstad, S. Einfeldt, D. Hommel, T. Berge and A. Grande, J. Appl. Phys 83, 6023 (1998).

[3] M. E. Lin, B. N. Sverdlov, and H. Morkoç, J. Appl. Phys. 74, 5038 (1993).

[4] T. D. Moustakas, T. Lei, and R. J. Molnar, Physica B 185, 36 (1993).

[5] R. J. Molnar and T. D. Moustakas, J. Appl. Phys. 76, 4587 (1994).

[6] I. Berishev, E. Kim and A. bensaoula, J. Vac. Sci. Technol. A16, 2791 (1998).

[7] Handbook of Auger Electron Spectroscopy, Perkin-Elmer, Physical Electronics Division (Eden Prairie, MN, 1995).

[8] M. Yeadon, M. T. Marshall, F. Hamdani, S. Pekin, and H. Morkoç, J. Appl. Phys. 83, 2847 (1998).

[9] Analysis followed standard procedures such as outlined in D. Briggs and M.P. Seah, Practical Surface Analysis Vol. 1, John Wiley and Sons (Chichester, England, 1990), p. 207.

[10] S. Tanuma, C.J. Powell, and D.R. Penn, Surf. Interface Anal., 17911 (1991).

[11] M. Moldovan, L.S. Hirsch, A.J. Ptak, C.D. Stinespring, T.H. Myers, and N.C. Giles, J. Elec. Mat, 27, 756 (1998). 